\title{
Factors associated with increased irisin levels in the type 1 diabetes mellitus
}

\author{
${ }^{1}$ Ates I, ${ }^{1}$ Arikan MF, ${ }^{1}$ Erdogan K, ${ }^{1}$ Kaplan M, ${ }^{2}$ Yuksel M, ${ }^{3}$ Topcuoglu C, ${ }^{1}$ Yilmaz N, ${ }^{4,5}$ Guler S
}

${ }^{1}$ Ankara Numune Training and Research Hospital, Department of Internal Medicine, Ankara, Turkey; ${ }^{2}$ Turkey Yuksek Ihtisas Training and Research Hospital, Department of Gastroenterology, Ankara, Turkey;

${ }^{3}$ Ankara Numune Training and Research Hospital, Department of Biochemistry, Ankara, Turkey;

${ }^{4}$ Ankara Numune Training and Research Hospital, Department of Biochemistry, Ankara, Turkey; ${ }^{5}$ Hitit University School of Medicine, Department of Endocrinology and Metabolism, Çorum, Turkey E-mail:dr.ihsanates@hotmail.com

Objective. The aim of the present study was to determine the irisin levels in patients with the type 1 diabetes mellitus (T1DM) and to examine the relation of irisin levels with the inflammation and autoimmunity.

Methods. This study included 35 cases diagnosed with T1DM and 36 healthy volunteers. Antiglutamic acid decarboxylase (anti-GAD), islet cell antibody (ICA), and insulin autoantibody levels were measured in patients at the time when they were included into the study and recorded from the patient files. Serum irisin levels were measured by ELISA kit.

Results. The median irisin levels were determined higher in T1DM group compared to the control one $(6.8 \mathrm{ng} / \mathrm{ml}$ vs. $4.8 \mathrm{ng} / \mathrm{ml}, \mathrm{p}=0.022$; respectively). Median irisin levels were higher in anti-GAD $(\mathrm{p}=0.022)$ and ICA $(\mathrm{p}=0.044)$ positive groups compared to negative groups. In T1DM group, irisin levels displayed positive correlation with glycosylated hemoglobin (HbA1c) $(r=0.377$, $\mathrm{p}<0.001)$ and anti-GAD $(\mathrm{r}=0.392, \mathrm{p}=0.020)$ and negative correlation with creatinine $(\mathrm{r}=-0390$, $\mathrm{p}=0.021)$. In multivariate regression model, HbA1c $(\mathrm{B} \pm \mathrm{SE}: 2.76 \pm 17683, \mathrm{p}<0.001)$, and anti-GAD ( $\mathrm{B} \pm \mathrm{SE}: 2.311 \pm 0.610, \mathrm{p}=0.001)$ were determined as independent predictors for predicting the irisin levels.

Conclusion. In patients with T1DM, which chronic inflammation and autoimmunity take part in their etiopathogenesis, anti-GAD levels were an independent risk factor for the irisin. This may suggest that factors such as inflammation and autoimmunity can be effective in the synthesis of irisin.

Key words: IAnti-glutamic acid decarboxylase, fibronectin type III domain containing 5, HbA1c, hyperglycemia, PGC1- $a$

Irisin, being discovered in 2012, is a myokine, synthesized majorly in the skeletal muscle (Bostrom et al. 2012). Although according to recent studies, the primary factor affecting the synthesis of irisin is considered to be exercise, different mechanisms may play a role in the synthesis of irisin. After exercising, the synthesis of fibronectin type III domain containing 5 (FNDC5) increases in the skeletal muscle with the activation of peroxisome proliferator-activated receptor $\gamma$ (PPAR $\gamma)$ coactivator 1a. Then FNDC5 is broken down to irisin, majorly in the skeletal muscle, with an unknown protease enzyme (Ates et al. 2016; Aydin

Corresponding author: Dr. Ihsan Ates, MD, Ankara Numune Training and Research Hospital, Department of Internal Medicine, 06100, Sihhiye, Ankara, Turkey; phone: +90 312 5084666; fax: +90 31235690 03; e-mail: dr.ihsanates@hotmail.com. 
2014). Irisin turns the white adipose tissue to brown one and causes thermogenesis (Bostrom et al. 2012). White adipose tissue contains triglyceride and fatty acid as well as a small amount of mitochondria (De Naeyer et al. 2011). Brown adipose tissue contains more mitochondria compared to white adipose tissue (Enerback 2010). Therefore, while white adipose tissue acts as a lipid storage, brown adipose tissue plays a role in the energy expenditure. The uncoupling protein-1 (UCP-1), found in the mitochondria of brown adipose tissue that increases with the effect of irisin, causes heat buildup by pumping protons to mitochondrial matrix through the intermembrane space (Jastroch et al. 2010). Thus, energy consumption increases.

Due to these major effects of irisin on the metabolism, many studies have been previously conducted to investigate the association between irisin and metabolic diseases. Irisin levels have often been determined to be decreased in the type 2 diabetes mellitus (T2DM) (Choi et al. 2013) and increased in obesity (Crujeiras et al. 2014) and insulin resistance (Staiger et al. 2013). It has recently been reported that irisin levels increase in T1DM patients (Espes et al. 2015). In most of these studies, insulin metabolism has been shown to be effective in the variation of irisin levels. We could not find any data indicating how the insulin levels are affected by inflammation and autoimmunity. However, since irisin is a myokine synthesized based on the peroxisome proliferator-activated receptor gamma coactivator 1 -alpha (PGC-1 $\alpha$ ), we think that it is also associated with inflammation. Because it is known that PGC-1 $\alpha$ by increasing the synthesis of proinflammatory cytokines may increase the systemic inflammation at the same time (Handschin 2009).

Therefore, we aimed to determine the irisin level in patients with T1DM, in whom both chronic inflammation and autoimmunity play a role in its etiopathogenesis and to examine the relation of irisin levels with inflammation and autoimmunity.

\section{Material and Methods}

Study population. This study was conducted in Ankara Numune Training and Research Hospital (ANEAH), Clinic of Internal Medicine between May and September 2015.

The study included a total of 71 adult participants consisting of 35 cases (16 males, 19 females) diagnosed with T1DM and 36 healthy volunteers (15 males, 21 females). T1DM group consisted of patients with documented diagnosis of T1DM, who admitted to our clinic for routine control. The healthy control group consisted of voluntary participants who admitted to our hospital for check-up or other reasons, without any known chronic disease and drug use. When cases were being selected for the control group, they were taken respectively, regarding their age, sex, body mass index (BMI) to be similar to patients. BMI was calculated by dividing body dry weight to the square of tall stature in meters (BMI: $\mathrm{kg} / \mathrm{m}^{2}$ ).

Participants with known T2DM, documented cardiovascular and cerebrovascular diseases, liver and kidney failure, thyroid disorders, malignancy, inflammatory diseases related to rheumatism or infections, malnutrition, morbid obesity, smoking, alcohol and drug consumption and those doing regular exercises were excluded from the study. Also patients with high (>100 mg/dl) fasting blood glucose (FBG) in the control group were excluded.

This study was designed in accordance with the Helsinki Declaration and approved by the Research Ethics Committee of ANEAH. All participants provided written informed consent.

Biochemical parameters. To determine the irisin levels of all participants who were included in the study, venous blood samples were drawn between 08:00-10:00 a.m. after 8 h of fasting. After blood samples were swiftly centrifuged $4000 \mathrm{rpm}$ for $10 \mathrm{~min}$, plasma was separated and serum samples were stored at $-80^{\circ} \mathrm{C}$. Then in all samples, the irisin levels were studied in the same session.

Fasting blood glucose and creatinine were measured with enzymatic colorimetric method, C-reactive protein (CRP) by immunoturbidimetric method, C-peptide level with chemiluminescence immunoassay method in Hitachi Modular P800 auto analyzer (Roche Diagnostic Corp. Indianapolis, Indiana, USA). Glycosylated hemoglobin (HbAlc) was measured with cation exchange high-performance liquid chromatography method by using Arkray ADAMS Alc HA8180 Alc automatic glycohemoglobin analyzer (Arkray Global Business, Inc., Kyoto. Japans).

Serum irisin levels were measured by commercial ELISA kit (EASTBIOPHARM, Hangzhou Eastbiopharm Co. Ltd. China, REF: CK-E90905, LOT: 201,402). Intra-study CV\% $<10$ and inter-study \%CV $<12$.

Anti-glutamic acid decarboxylase (anti-GAD), islet cell antibody (ICA), insulin autoantibody levels of the patients were measured at the time when they were included in the study and were recorded from the patient files. Anti-GAD, ICA and insulin autoantibody levels were measured with a Cobas e 601 autoanalyzer (Catolog No. 11731459124, 03051986192, 1173129724, 
Roche Diagnostics, Mannhaim, Germany) using the electrochemiluminescence immunoassay method.

Statistical Analysis. Statistical analyses were performed using Statistical Package for Social Sciences for Windows 20 program (IBM SPSS Inc., Chicago, IL). Normal distribution of data was evaluated with Kolmogorov-Smirnov test. Numerical variables showing normal distribution were expressed as mean \pm standard deviation (SD) and those not showing normal distribution were expressed as median [interquartile range]. Categorical variables were expressed as numbers and percentage. Student's t test and Mann-Whitney U test were used to compare two groups of numerical variables. Chi-square and Fisher's Exact tests were used to compare categorical variables. The relationship between numerical variables was evaluated using Pearson and Spearman correlation analysis. Risk factors that might be effective on irisin levels were determined with multivariate linear regression analysis. Value $\mathrm{p}<0.05$ was considered statistically significant.

\section{Results}

Table 1 summarizes the demographic characteristics and laboratory findings of study population. Sex distribution $(\mathrm{p}=0.813)$, mean age $(\mathrm{p}=0.732)$ and BMI ( $\mathrm{p}=0.492)$ levels were similar in both groups. The mean age of the women in T1DM group was higher than that of men (36.8 years vs. 21.5 years, $\mathrm{p}<0.05$, respectively). The median duration of diabetes in T1DM patients were 3 years. Median HbAlc $(81 \mathrm{mmol} / \mathrm{mol}$ vs. $30 \mathrm{mmol} / \mathrm{mol}, \mathrm{p}<0.001$, respectively) and FBG (205 mg/dl vs. $89 \mathrm{mg} / \mathrm{dl}, \mathrm{p}<0.001$, respectively) levels were higher in T1DM patients compared to the control group. While median creatinine levels were similar between the two groups, CRP levels ( $3.4 \pm 0.8 \mathrm{mg} / \mathrm{l}$ vs. $1.2 \pm 0.6 \mathrm{mg} / \mathrm{l}, \mathrm{p}<0.001$, respectively) were higher in the patient group. In T1DM group, the median irisin level $(6.8 \mathrm{ng} / \mathrm{ml}$ vs. $4.8 \mathrm{ng} / \mathrm{ml}, \mathrm{p}=0.022$, respectively) was higher than in the control group (Figure 1), the mean C-peptide level $(0.4 \pm 0.1 \mathrm{ng} / \mathrm{ml}$ vs. $1.8 \pm 0.1 \mathrm{ng} / \mathrm{ml}, \mathrm{p}<0.001$, respectively) was lower compared to the control group.

When irisin levels were examined in T1DM patients according to presence of antibody; they were found higher in anti-GAD ( $\mathrm{p}=0.022)$ positive and ICA ( $\mathrm{p}=0.044)$ positive groups compared to negative groups. As for IAA group, there was no significant change in irisin levels based on antibody presence $(\mathrm{p}=0.674)$ (Table 2). When irisin levels were examined according to sex distribution, similar levels between the male and female groups were obtained ( $p>0.05)$.
In Table 3, the parameters associated with irisin levels are shown in the correlation analysis. In T1DM group irisin levels displayed a positive correlation with $\mathrm{HbAlc}(\mathrm{r}=0.377, \mathrm{p}<0.001)$ and anti-GAD

Table 1

Demographic characteristics and laboratory findings of study populations

\begin{tabular}{lccc}
\hline Variables & $\begin{array}{c}\text { T1DM } \\
\mathbf{n}(35)\end{array}$ & $\begin{array}{c}\text { Control } \\
\mathbf{n}(36)\end{array}$ & p-value \\
\hline Gender, n (\%) & & & \\
Female & $19(54.3)$ & $21(58.3)$ & 0.813 \\
Male & $16(45.7)$ & $15(41.7)$ & \\
Age (years) & $29.4 \pm 9.2$ & $28.7 \pm 8.1$ & 0.732 \\
BMI (kg/m²) & $25.6 \pm 5.2$ & $24.8 \pm 4.6$ & 0.492 \\
DM duration & $3(17)$ & - & - \\
HbAlc $(\%)$ & $9.6(2.5)$ & $4.9(0.4)$ & $<0.001^{*}$ \\
FBG (mg/dl) & $205(147)$ & $89(3.8)$ & $<0.001^{*}$ \\
Creatinine (mg/dl) & $0.7(0.1)$ & $0.7(0.1)$ & 0.977 \\
CRP (mg/l) & $3.4 \pm 0.8$ & $1.2 \pm 0.6$ & $<0.001^{*}$ \\
\hline Irisin (ng/ml) & $6.8(44.4)$ & $4.8(4.7)$ & $0.022^{*}$ \\
C-peptide (ng/ml) & $0.4 \pm 0.1$ & $1.8 \pm 0.1$ & $<0.001^{*}$ \\
\hline
\end{tabular}

Quantitative variables are shown as mean \pm standard deviation or median. Categorical variables are shown as numbers (\%). ${ }^{*} \mathrm{p}<0.05$ statistical significance

Abbreviations: T1DM - type 1 diabetes mellitus; BMI - body mass index; HbA1C - glycosylated hemoglobin; FBG - fasting blood glucose; CRP - c-reactive protein

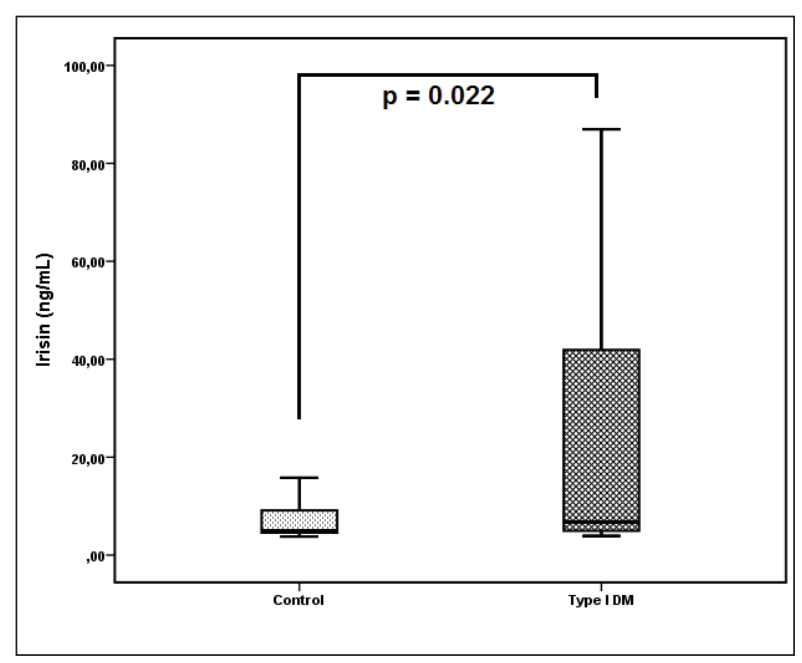

Figure 1. Irisin level between the type 1 diabetes mellitus and control groups. 
$(\mathrm{r}=0.392, \mathrm{p}=0.020)$ (Figure 2$)$ and a negative correlation with creatinine $(\mathrm{r}=-0390, \mathrm{p}=0.021)$. There was no significant relation determined between the other parameters and irisin levels.

When the effects of age, sex, BMI, and disease duration are cleansed, $\mathrm{HbAlc}$ and anti-GAD levels were single risk factors effective on the irisin levels while the impact of creatinine level lost its significance. In the multivariate regression model that included risk factors such as age, sex, BMI, disease duration,

Table 2

The change in irisin level in type 1 diabetes mellitus patients according to antibody presence

\begin{tabular}{lccc}
\hline Variables & Groups & $\begin{array}{c}\text { Irisin level } \\
(\mathbf{n g} / \mathbf{m l})\end{array}$ & p-value \\
\hline Anti-GAD & Negative $(\mathrm{n}=14)$ & $4.9(4.7)$ & $0.022^{*}$ \\
& Positive $(\mathrm{n}=21)$ & $6.4(7.3)$ & \\
\hline ICA & Negative $(\mathrm{n}=27)$ & $6.7(16.2)$ & $0.044^{*}$ \\
& Positive $(\mathrm{n}=8)$ & $27.0(163.8)$ & \\
& Negative $(\mathrm{n}=13)$ & $6.7(36.9)$ & \\
\multirow{2}{*}{ IAA } & Positive $(\mathrm{n}=22)$ & $6.9(81.3)$ & 0.674 \\
\hline
\end{tabular}

Quantitative variables are shown with median. ${ }^{\star} \mathrm{p}<0.05$ statistical significance.

Abbreviations: Anti-GAD - anti-glutamic acid decarboxylase; ICA - islet cell antibody; IAA - insulin antibody

Table 3

Irisin level in type 1 diabetes mellitus patients and the association between clinical and laboratory findings

\begin{tabular}{lcc}
\hline \multirow{2}{*}{ Variables } & \multicolumn{2}{c}{ Irisin } \\
\cline { 2 - 3 } Age & 0.120 & p-value \\
\hline BMI & -0.055 & 0.492 \\
DM duration & -0.137 & 0.753 \\
\hline HbA1c & 0.377 & 0.432 \\
FBG & -0.140 & $<0.001^{*}$ \\
\hline Anti-GAD & 0.392 & 0.422 \\
\hline IAA & 0.187 & $0.020^{*}$ \\
Creatinine & -0.390 & 0.283 \\
CRP & 0.264 & $0.021^{*}$ \\
C-peptide & 0.071 & 0.125 \\
\hline
\end{tabular}

${ }^{*} \mathrm{p}<0.05$ statistical significance

Abbreviations: DM - diabetes mellitus; BMI - body mass index; HgA1C - hemoglobin A1c; FBG - fasting blood glucose; CRP c-reactive protein
HbAlc, FBG, anti-GAD, IAA, creatinine, CRP and C-peptide (B \pm SE: $17683 \pm 2.76, \mathrm{p}<0.001), \mathrm{HbAlc}$ and anti-GAD (B \pm SE: $2.311 \pm 0.610, p=0.001)$ were determined as independent predictors for predicting the irisin levels (Table 4).

\section{Discussion}

In the present study, irisin levels were determined higher in the T1DM patients compared to the control group. In anti-GAD and ICA positive patients, irisin levels were found higher than in negative patients. Irisin levels displayed a positive correlation with $\mathrm{HbAlc}$ and anti-GAD in T1DM patients. In multivariable regression analysis, HbAlc and anti-GAD were determined to independent risk factors for irisin levels. This is the first study examining the relation of irisin levels with autoimmunity in T1DM patients.

Irisin is a recently discovered myokine that is thought to be associated with the metabolic diseases. While at the beginning, it was considered to be a hormone that can only be synthesized in skeletal muscle, it was later found to be synthesized in many tissues in the body (Huh et al. 2012). It is believed to be primarily associated with the exercises. It shows its effects by influencing UCP-1's found in the mitochondria of adipose tissue. UCP-1 increases heat generation by pumping protons coming from the electron transport chain to mitochondrial matrix through intermembrane space and blocking the formation of adenosine triphosphate (Affourtit et al. 2012). With these mechanisms, irisin hormone appeared to be anti-obesity hormone. Indeed, the studies conducted

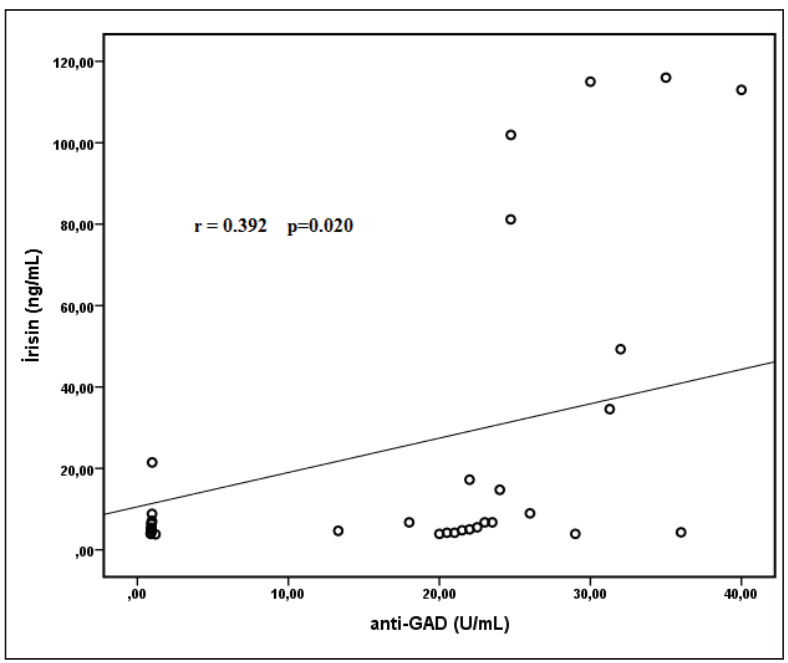

Figure 2. Correlation analysis between the irisin and antiGAD. 
Table 4

Risk factors affecting irisin level in type 1 diabetes mellitus patients

\begin{tabular}{|c|c|c|c|c|c|c|c|c|c|c|c|c|}
\hline \multirow{3}{*}{ Variables } & \multicolumn{4}{|c|}{ Unadjusted } & \multicolumn{4}{|c|}{ Adjusted $^{\ddagger}$ Univariable } & \multicolumn{4}{|c|}{ Adjusted $^{\bigotimes}$ Multivariable } \\
\hline & \multirow{2}{*}{$\mathrm{B} \pm \mathrm{SE}$} & \multicolumn{2}{|c|}{ 95\% C.I. } & \multirow{2}{*}{ p-value } & \multirow{2}{*}{$\mathrm{B} \pm \mathrm{SE}$} & \multicolumn{2}{|c|}{ 95\% C.I. } & \multirow{2}{*}{ p-value } & \multirow{2}{*}{$\mathrm{B} \pm \mathrm{SE}$} & \multicolumn{2}{|c|}{ 95\% C.I. } & \multirow{2}{*}{ p-value } \\
\hline & & lower & upper & & & lower & upper & & & lower & upper & \\
\hline $\mathrm{HbAlc}$ & $1.629 \pm 0.66$ & 0.276 & 2.982 & $0.020^{*}$ & $2.300 \pm 0.98$ & 0.298 & 4.302 & $0.026^{\star}$ & $2.311 \pm 0.610$ & 1.068 & 3.554 & $0.001^{*}$ \\
\hline FBG & $0.006 \pm 0.06$ & -0.113 & 0.126 & 0.918 & $0.027 \pm 0.06$ & -0.098 & 0.153 & 0.666 & $-0.013 \pm 0.05$ & -0.123 & 0.098 & 0.814 \\
\hline Anti-GAD & $2.427 \pm 0.54$ & 1.351 & 3.500 & $<0.001^{*}$ & $2.875 \pm 0.56$ & 1.752 & 3,997 & $<0.001^{*}$ & $2.947 \pm 0.46$ & 1.993 & 3,901 & $<0.001^{\star}$ \\
\hline IAA & $1.137 \pm 1.04$ & -0.980 & 3.253 & 0.283 & $0.486 \pm 0.971$ & -1.500 & 2.471 & 0.621 & $-0.393 \pm 0.72$ & -1.894 & 1.108 & 0.593 \\
\hline Creatinine & $-1.118 \pm 0.51$ & -2.200 & -0.162 & $0.035^{\star}$ & $-13.35 \pm 12.75$ & -38.824 & 12.125 & 0.299 & $-12.789 \pm 8.41$ & -30.188 & 4.609 & 0.142 \\
\hline CRP & $-0.012 \pm 0.13$ & -0.289 & 0.265 & 0.930 & $-0.033 \pm 0.134$ & -0.308 & 0.242 & 0.807 & $0.016 \pm 0.09$ & -0.180 & 0.211 & 0.869 \\
\hline \multirow[t]{2}{*}{ C-peptide } & $6.181 \pm 16.97$ & -28.346 & 40.709 & 0.683 & $-5.446 \pm 60.55$ & -41.193 & 30.302 & 0.758 & $-7.395 \pm 13.54$ & -35.412 & 20.621 & 0.590 \\
\hline & & & & & & & & & \multicolumn{4}{|c|}{ Adj $R^{2}=0.344 . p<0.001^{*}$} \\
\hline
\end{tabular}

${ }^{\ddagger}$ Age, sex, BMI and disease duration are adjusted

Abbreviations: DM - diabetes mellitus; BMI - body mass index; HbA1C - glycosylated hemoglobin A1C; FBG - fasting blood glucose; Anti-GAD - anti-glutamic acid decarboxylase; IAA - insulin auto-antibody; CRP - C-reactive protein

later have determined that irisin levels were higher in the obese patients compared to the healthy control group (Crujeiras et al. 2014).

The association between T2DM and iris levels is often being questioned in recent studies. Studies have shown that irisin levels were lower in the T2DM patients compared to the control ones (Choi et al. 2013; Moreno-Navarrete et al. 2013; Liu et al. 2014). In studies conducted with patients with insulin resistance, irisin levels were determined to increase with the insulin resistance and decrease as insulin sensitivity increases (Sesti et al. 2014). In a study conducted with patients with T1DM, which inflammation and autoimmunity play a role in its etiopathogenesis, irisin levels were found to be higher compared to the control group (Espes et al. 2015). In the same study, irisin levels were found to be lower in men compared to women. Considering the results of these studies, it is possible to conclude that irisin hormone is not only associated with exercise, but also with hormones, insulin resistance, inflammation, and autoimmunity.

In our study, irisin levels were determined higher in the TIDM patients compared to the control ones. The data of this study were similar to the other study conducted with T1DM patients. But while irisin levels differed according to the sex in the other study, there was no such difference determined in our study (Espes et al. 2015). This situation can be explained by the age and BMI. In the other study, BMI levels of women were higher than the men (although not statistically significant). The positive correlation be- tween BMI and irisin has been shown in previous studies (Park et al. 2014). As for its relation with age, the mean age of women in T1DM group of our study was fairly high compared to the other study. Age and irisin levels displayed a negative correlation in the other study (Espes et al. 2015). These results clarify why irisin levels did not differ between different sexes in our study.

In the correlation analysis of our study, irisin levels were determined to be negatively correlated with creatinine and positively correlated with $\mathrm{HbAlc}$ and anti-GAD. In a previous study conducted with patients with chronic renal failure, irisin levels were determined to be negatively correlated with creatinine (Wen et al. 2013). This case is considered to result from the inhibition of FNDC5, which is the precursor of irisin, by indoxyl sulphate, which is a uremic toxin (Wen et al. 2013). We did not found any study examining the relation between irisin and HbAlc in T1DM patients. However, in studies conducted with patients with the T2DM and metabolic syndrome in contrast with our study - irisin levels were negatively correlated with HbAlc (Choi et al. 2013; Yan et al. 2014). This relation between irisin and HbAlc have not been interpreted in these studies. Since HbAlc is an indirect indicator of hyperglycemia, the determination of a positive correlation between irisin and $\mathrm{HbAlc}$ is not a surprising finding. Because previous studies have shown that there is a positive correlation between the blood glucose level and irisin (Liu et al. 2013). In addition, another study has suggested that 
irisin increases as a compensatory answer for hyperglycemia (Crujeiras et al. 2014). Also the determination of HbAlc as an independent risk factor for irisin, in the multivariate regression analysis, supports our thesis.

The determination of the high irisin levels in antibody positive patients compared to negative ones and determination of a positive correlation between irisin levels and anti-GAD; suggests that autoimmunity may have a role in the increase of irisin levels. The relation between irisin and autoimmunity can be explained as follows: When the PGC-1 $\alpha$ levels increase due to exercise or any other cause; a chronic inflammatory situation may occur at the cellular level, with the increase in the level of cytokines such as IL-6, IL-8 and IL-15 from a pathway (in our study $\mathrm{CRP}$, an indicator of a chronic inflammation, was determined higher in the patient group) (Pedersen et al. 2003; Febbraio 2007). With this inflammation, occurring with the increase in $\mathrm{T}$ cell response, both the number and immunogenicity of auto-antibodies that play a role in autoimmune incidents, may increase. PGC-1 $\alpha$, through another pathway, increases FNDC5 level and leads to an increase in irisin release (Aydin 2014). Thus, both auto-antibody levels and irisin levels may be increased by the PGC-1 $\alpha$ activation. So far, our explanations support our results up to the correlation analysis part. However, this situation does not explain why anti-GAD is an independent risk factor for irisin. So we can bring a possible explanation such as: Auto-antibody levels that increase as a result of chronic inflammation, can lead to the release of current or synthesized irisin molecules in the pancreas by increasing pancreatic B cell destruction. Because in a study in which specific irisin antibody has been used to show irisin immunoreactivity in pancreas, irisin has been shown to exist in the center pancreatic cells, periphery of Langerhans islets, and capillary (Aydin 2014). Moreover, the presence of the irisin in pancreas has been shown in another study (Aydin et al. 2014). This can be an evidence that irisin is synthesized in pancreas and may be increased with the destruction of pancreas.

The main limitation of our study is its cross-sectional design and consequently not being able to follow patients to see how irisin levels differ with the changes in auto-antibody levels.

As a result, unlike other metabolic disorders, higher irisin levels were determined in T1DM patients compared to the control ones. This may suggest that factors such as inflammation and autoimmunity can be effective in the synthesis of irisin. To clarify the association between the autoimmunity and irisin levels previously conducted, randomized control studies, with more number of patients and a longer follow-up process, are required.

\section{References}

Affourtit C, Quinlan CL, Brand MD. Measurement of proton leak and electron leak in isolated mitochondria. Methods Mol Biol 810, 165-182, 2012.

Ates I, Altay M, Topcuoglu C, Yilmaz FM. Circulating levels of irisin is elevated in hypothyroidism, a case-control study. Arch Endocrinol Metab 60, 95-100, 2016.

Aydin S. Three new players in energy regulation: preptin, adropin and irisin. Peptides 56, 94-110, 2014.

Aydin S, Kuloglu T, Aydin S, Kalayci M, Yilmaz M, Cakmak T, Albayrak S, Gungor S, Colakoglu N, Ozercan IH. A comprehensive immunohistochemical examination of the distribution of the fat-burning protein irisin in biological tissues. Peptides 61, 130-136, 2014.

Bostrom P, Wu J, Jedrychowski MP, Korde A, Ye L, Lo JC, Rasbach KA, Bostrom EA, Choi JH, Long JZ, Kajimura S, Zingaretti MC, Vind BF, Tu H, Cinti S, Hojlund K, Gygi SP, Spiegelman BM. A PGC1-alpha-dependent myokine that drives brown-fat-like development of white fat and thermogenesis. Nature 481, 463-468, 2012.

Choi YK, Kim MK, Bae KH, Seo HA, Jeong JY, Lee WK, Kim JG, Lee IK, Park KG. Serum irisin levels in new-onset type 2 diabetes. Diabetes Res Clin Pract 100, 96-101, 2013.

Crujeiras AB, Pardo M, Arturo RR, Navas-Carretero S, Zulet MA, Martinez JA, Casanueva FF. Longitudinal variation of circulating irisin after an energy restriction-induced weight loss and following weight regain in obese men and women. Am J Hum Biol 26, 198-207, 2014.

De Naeyer H, Ouwens DM, Van Nieuwenhove Y, Pattyn P, Hart LM, Kaufman JM, Sell H, Eckel J, Cuvelier C, Taes YE, Ruige JB. Combined gene and protein expression of hormone-sensitive lipase and adipose triglyceride lipase, mitochondrial content, and adipocyte size in subcutaneous and visceral adipose tissue of morbidly obese men. Obes Facts 4, 407-416, 2011.

Enerback S. Brown adipose tissue in humans. Int J Obes (Lond) 34 Suppl 1, S43-46, 2010. 
Espes D, Lau J, Carlsson PO. Increased levels of irisin in people with long-standing Type 1 diabetes. Diabet Med 32, 1172-1176, 2015.

Febbraio MA. Exercise and inflammation. J Appl Physiol (1985) 103, 376-377, 2007.

Handschin C. Peroxisome proliferator-activated receptor-gamma coactivator-1alpha in muscle links metabolism to inflammation. Clin Exp Pharmacol Physiol 36, 1139-1143, 2009.

Huh JY, Panagiotou G, Mougios V, Brinkoetter M, Vamvini MT, Schneider BE, Mantzoros CS. FNDC5 and irisin in humans: I. Predictors of circulating concentrations in serum and plasma and II. mRNA expression and circulating concentrations in response to weight loss and exercise. Metabolism 61, 1725-1738, 2012.

Jastroch M, Divakaruni AS, Mookerjee S, Treberg JR, Brand MD. Mitochondrial proton and electron leaks. Essays Biochem 47, 53-67, 2010.

Liu JJ, Wong MD, Toy WC, Tan CS, Liu S, Ng XW, Tavintharan S, Sum CF, Lim SC. Lower circulating irisin is associated with type 2 diabetes mellitus. J Diabetes Complications 27, 365-369, 2013.

Liu JJ, Liu S, Wong MD, Tan CS, Tavintharan S, Sum CF, Lim SC. Relationship between circulating irisin, renal function and body composition in type 2 diabetes. J Diabetes Complications 28, 208-213, 2014.

Moreno-Navarrete JM, Ortega F, Serrano M, Guerra E, Pardo G, Tinahones F, Ricart W, Fernandez-Real JM. Irisin is expressed and produced by human muscle and adipose tissue in association with obesity and insulin resistance. J Clin Endocrinol Metab 98, E769-778, 2013.

Park KH, Zaichenko L, Peter P, Davis CR, Crowell JA, Mantzoros CS. Diet quality is associated with circulating Creactive protein but not irisin levels in humans. Metabolism 63, 233-241, 2014.

Pedersen BK, Steensberg A, Fischer C, Keller C, Keller P, Plomgaard P, Febbraio M, Saltin B. Searching for the exercise factor: is IL-6 a candidate? J Muscle Res Cell Motil 24, 113-119, 2003.

Sesti G, Andreozzi F, Fiorentino TV, Mannino GC, Sciacqua A, Marini MA, Perticone F. High circulating irisin levels are associated with insulin resistance and vascular atherosclerosis in a cohort of nondiabetic adult subjects. Acta Diabetol 51, 705-713, 2014.Staiger H, Bohm A, Scheler M, Berti L, Machann J, Schick F, Machicao F, Fritsche A, Stefan N, Weigert C, Krook A, Haring HU, de Angelis MH. Common genetic variation in the human FNDC5 locus, encoding the novel muscle-derived 'browning' factor irisin, determines insulin sensitivity. PLoS One 8, e61903, 2013.

Wen MS, Wang CY, Lin SL, Hung KC. Decrease in irisin in patients with chronic kidney disease. PLoS One 8, e64025, 2013.

Yan B, Shi X, Zhang H, Pan L, Ma Z, Liu S, Liu Y, Li X, Yang S, Li Z. Association of serum irisin with metabolic syndrome in obese Chinese adults. PLoS One 9, e94235, 2014. 